\title{
The association of single nucleotide polymorphisms in TNFAIP3 with rheumatoid arthritis in the Chinese population
}

\author{
XINGANG ZHANG ${ }^{l}$,WEI LI $I^{2}$ XINPENG ZHANG ${ }^{3}$, XIAOLI ZHANG ${ }^{l}, L_{I} J_{I A N G}^{l}, Y U N G U O^{l}$, \\ JIN ZHANG ${ }^{4}$, XIAOFEI WANG ${ }^{l}$, ZAIFU LIANG ${ }^{5}$
}

\begin{abstract}
Objective: Genome-wide association studies of rheumatoid arthritis (RA) in Caucasian populations have identified polymorphisms in and near the tumor necrosis factor $\alpha(T N F-\alpha)$-induced protein 3 gene (TNFAIP3). This study aimed to investigate the association between the critical polymorphisms in the TNFAIP3 gene and the risk of RA in the Chinese population.

Material and methods: A hospital-based case-control study of 344 RA patients and 344 matched controls was conducted. After informed consent was obtained, each person donated $2 \mathrm{ml}$ of blood for biomarker testing. Three polymorphisms were determined by the TaqMan SNP genotyping method. The statistical analyses were performed primarily with SPSS (v13.0).

Results: This study showed that individuals with the rs2230926 TG genotype had an increased risk of $R A$ compared with those carrying the TT genotype (OR $=1.74,95 \% C I: 1.12-2.72, p=0.015)$ and the combined TG/GG genotypes were also associated with an increased risk of $R A(O R=1.75,95 \% C I$ : 1.13-2.72, $p=0.012$ ). Individuals carrying the rs10499194 CT heterozygous genotype had a 1.88-fold increased risk of RA compared with those with the homozygous wild genotype CC (95\% CI: 1.05-3.36, $p=0.034)$. Subset analysis stratified to gender showed that significant associations were present in the female population, but not in the male population. Haplotype analysis showed that the T-A-C and G-G-C haplotypes were associated with increased risks of RA (ORs were 2.46 and 2.27, 95\% CIs were 1.36-4.48 and 1.35-3.80, $p$ values were 0.002 and 0.002 , respectively).

Conclusions: The rs 10499194 and rs2230926 polymorphisms in the TNFAIP3 gene region may be genetic risk modifiers for $R A$ in the Chinese population.
\end{abstract}

Key words: TNFAIP3 gene, single nucleotide polymorphism, rheumatoid arthritis.

(Centr Eur J Immunol 2013; 38 (2): 214-220)

\section{Introduction}

Rheumatoid arthritis (RA) is an autoimmune inflammatory disease involving inflammation and destruction of synovial joints, which leads to progressive joint damage and disability. RA is the most common type of inflammatory arthritis and as much as $1 \%$ of the worldwide population is affected by RA [1]. Rheumatoid arthritis is a multi- genic disease and is believed to be caused by interactions between susceptible genes and environmental factors. Although the etiology and risk factors for RA are not fully understood, it is known that strong genetic susceptibility is an important component of this disease [2]. Familial and linkage studies provide strong evidence for the role of genetic factors in the development of RA [3]. In addition, population-based association studies about single nucleotide

Correspondence (equal contributions): Zaifu Liang, Xiaofei Wang, Shenyang, Liaoning Province, China, tel. (86)18940257526, fax (86)024-23926295, e-mail: 18940257526@189.cn 
polymorphisms (SNPs) in candidate loci have also identified some genes that modify susceptibility to RA. More recently, genome-wide association studies (GWAS) in RA have discovered susceptibility genes that contribute to the development of RA [4, 5]. Recent GWAS in Caucasian populations have identified the tumor necrosis factor $\alpha$ (TNF- $\alpha$ )-induced protein 3 gene (TNFAIP3) as a common genetic risk factor for RA $[6,7]$. TNFAIP3, located at 6q23, encodes a ubiquitin-editing protein, A20. This protein is required for the termination of TNF and is known as a negative regulator of the nuclear factor- $\kappa \mathrm{B}(\mathrm{NF}-\kappa \mathrm{B})$ signaling pathway, which is essential in the pathogenesis of RA $[8,9]$. Furthermore, another study showed that A20-deficient mice could develop severe inflammation, including inflammation of the joints [10].

There are studies reporting that TNFAIP3 might be a susceptibility gene for RA in Caucasian populations $[6,7,11]$. Two SNPs in 6q23, namely rs6920220 and rs13207033, have been suggested to be associated with RA risks in Caucasian population [12]. Recently, Shimane replicated the association of TNFAIP3 SNPs and RA in a Japanese population [13]. They observed a significant association between rs2230926 and an increased risk of RA, as well as the association between rs10499194 and RA risk while the risk allele for RA in Caucasians was protective against the diseases in our population. There are three SNPs analyzed in the present study, among which rs6920220 and rs 13207033 are located in the intergenic region between TNFAIP3 and the oligodendrocyte transcription factor 3 gene (OLIG3), while rs2230926 is located in the TNFAIP3 gene.

No association between polymorphisms in the TNFAIP3 gene and RA risk in a Chinese population has been reported to date. In the present investigation, we describe a casecontrol study of RA in Shenyang, China, to evaluate the role of the polymorphisms of the TNFAIP3 gene on the risk of RA and to explore the interactions of these genetic polymorphisms in the development of RA.

\section{Material and methods}

\section{Study subjects and data collection}

In this hospital-based case-control study, conducted between July 2010 and June 2011, the case group consisted of 344 diagnosed RA patients. All RA cases fulfilled the diagnostic features of RA established by the American College of Rheumatology criteria [14]. At the same time, 344 unrelated healthy control subjects without any indication of RA were selected from the same hospital. Controls were all frequency matched to cases for age ( \pm 5 years) and gender. The human investigations were approved by the Ethics Committee of Shengjing Hospital of China Medical University (permission code 2010056), and informed consent was obtained from each participant. All patients were unrelated ethnic Han Chinese. Each participant donated $2 \mathrm{ml}$ of venous blood and was interviewed for the collection of demographic data at the time they were admitted to the hospital.

\section{Single nucleotide polymorphisms selection}

For the selection of SNPs in and near TNFAIP3, we reviewed three previous GWASs of RA $[6,7,15]$. These studies revealed that rs10499194, rs2230926 and rs6920220, which are located between TNFAIP3 and OLIG3, were significantly associated with the risk of RA. The HapMap data for Chinese individuals indicate that the minor allele frequency (MAF) of rs6920220 is $<0.001$. Results of a recent study in Korean populations also indicated that the variant was too rare $(\mathrm{MAF}<0.01)$ to be evaluated for associations [16]. Therefore, we selected SNPs rs10499194 and rs2230926 for evaluation. In addition, we reviewed a published study on the association between polymorphisms of the TNFAIP3 gene and the risk of RA in Asian populations, and another significant SNP, rs13207033, was selected for evaluation in the present study [13]. Thus, in total, three SNPs were analyzed in the present study: rs10499194, rs2230926 and rs13207033.

\section{DNA isolation and genotyping}

Genomic DNA samples were isolated by the phenolchloroform method. SNPs were analyzed by TaqMan assays. For the selected SNPs, predesigned TaqMan SNP genotyping assays were used. Primers and probes were synthesized by Shanghai Generay Biotechnology Co, Ltd. (P.R. China). The sequences of probes and primers are listed in Table 1. Fluorescence was detected using an ABI Prism 7500FAST Sequence Detection System (Applied Biosystems). Genotyping assessment was performed in $>98 \%$ of the samples for each of the polymorphisms genotyped. A 10\% masked random sample of patients was tested twice by different persons, and the results were found to be in agreement for all of the duplicate sets.

\section{Statistical analysis}

All statistical analyses were performed with SPSS (v13.0), if not otherwise specified. All of the tests were twosided, and statistical significance was defined as $p<0.05$. Pearson's $\chi^{2}$ test was used to compare the distribution of the demographic variables and examine differences in risk factors and genotypes, alleles and haplotypes between cases and controls. Hardy-Weinberg equilibrium (HWE) of the genotypes was tested by performing a goodness-of-fit $\chi^{2}$ test. Unconditional logistic regression analysis was performed to calculate the odds ratios (OR) with $95 \%$ confidence intervals (CI) for estimating the association between certain genotypes and RA. The interactions were evaluated by logistic regression models. On the basis of the observed frequencies of the three SNPs, we used the SHEsis analysis platform to calculate linkage disequilibrium indices (D' and $\left.\mathrm{r}^{2}\right)$ and infer haplotype frequencies $[17,18]$. 
Table 1. Sequences of Taqman probes and primers

\begin{tabular}{lll}
\hline SNP & Name & Sequence \\
\hline rs2230926 & forward primer & GATTTGAGAGACTCCAGTTGCCA \\
\hline & reverse primer & GCGTTCAGGACACAGACTTGG \\
\hline probe 1 & FAM-AGCGTGCTGAACAGCGCCTTC-TAMRA \\
\hline rs13207033 & probe 2 & HEX-AGCGTGCTGCACAGCGCCT-TAMRA \\
\hline & reverse primer & ATCAACATTTgTCTATTTTATgCTCCA \\
\hline probe 1 & FAM-TACAgATTTCACTTTCCCT-MgB \\
\hline & probe 2 & HEX-CTACAgATTTTACTTTCC-MGB \\
\hline forward primer & gCTATCAgTTTCATTACCTAAgAAATAgAgAC \\
\hline reverse primer & CAA Cgg ATA AgC AAT CAg ACC A \\
\hline probe 1 & FAM-AATgTgTTCAACCCTTT-MGB \\
\hline
\end{tabular}

SNP - single nucleotide polymorphism

\section{Results}

In total, 344 RA patients and 344 healthy controls were included in the present study. There were $288(83.7 \%)$ females and $56(16.3 \%)$ males in the case group, and 281 $(81.7 \%)$ females and $63(18.3 \%)$ males in the control group. The mean ages of the cases and controls (mean \pm SD) were almost identical (54.01 \pm 13.63 and $52.62 \pm 9.98$, respectively). There were no significant differences in the distributions of gender or age between the case and control groups.

Tables 2 and 3 present the distribution of genotypes and alleles for polymorphisms in the TNFAIP3 gene in cases and controls. The frequencies of the rs $2230926 \mathrm{G}$ allele, rs13207033 A allele and rs10499194 T allele were 0.055, $0.099,0.028$, respectively, in the controls and 0.090, 0.100, 0.049 , respectively, in the cases. All allele distributions were consistent with HWE ( $P$ values were $0.959,0.699,0.598$ in controls and $0.602,0.382,0.335$ in cases for the SNPs, respectively).

Among these SNPs, heterozygous carriers of the rs2230926 TG genotype had a 1.74-fold risk of RA compared with the homozygous wild genotype TT (95\% CI: $1.12-2.72, p=0.015)$. Individuals carrying the rs 10499194 CT heterozygous genotype had a 1.88-fold increased risk of RA compared with the homozygous wild genotype CC (95\% CI: $1.05-3.36, p=0.034)$. The rs 13207033 polymorphism was not associated with a risk of RA in this study. Considering the small sample size, further analyses were conducted by combining the heterozygous variant genotype with the homozygous variant genotype to evaluate these polymorphisms. As a result, the combined rs2230926 TG/GG was associated with an increased risk of RA $(\mathrm{OR}=1.75,95 \% \mathrm{CI}: 1.13-2.72, p=0.012)$.
Our results revealed a significant association between the risk of RA and both rs2230926 and rs10499194, when comparing allele frequency in patients and control subjects (ORs $=1.69$ and $1.83, p=0.013$ and 0.036 , respectively) (Table 3).

Additionally, we conducted a subset analysis stratified to gender. In the stratified analyses, we found that the increased risks associated with rs2230926 variant genotypes (TG) and rs10499194 variant genotypes (CT) were significant in the female population. The corresponding ORs were 1.66 (95\% CI: 1.02-2.69) and 1.89 (95\% CI: 1.04-3.44), respectively. Significant associations were not seen in the male population (Table 4). For allele analysis, we found that the $\mathrm{G}$ allele of rs2230926 and the T allele of rs10499194 were risk alleles for RA in the female population (ORs = $=1.62$ and 1.84 , 95\% CIs: $1.04-2.55$ and 1.02-3.30, respectively). Males carrying the $\mathrm{G}$ allele of rs2230926, the A allele of rs13207033 or the T allele of rs10499194 were also more likely to develop RA, but the results were not statistically significant. Although the sample size of subset analyses was too small for meaningful interpretation, especially for the male population, our results provided the baseline data of two genders. As we know, the genetic susceptibility of the disease could be different in two genders, thus it is an important idea to analyze the associated SNPs in male and female populations.

We evaluated the interaction of rs2230926 and rs10499194 on the risk of RA using a logistic regression model (Table 5). The results suggested that individuals with both risk genotypes of the two SNPs have a higher elevated risk of RA than those with only one of them $(\mathrm{OR}=4.44$, compared to 1.53 and 1.57 ).

We analyzed haplotypes using the SHEsis program platform (Table 6). The three SNPs were in linkage disequilib- 
Table 2. Association study of polymorphisms in the TNFAIP3 gene with rheumatoid arthritis in Chinese subjects

\begin{tabular}{|c|c|c|c|c|}
\hline Genotype & Cases $(\%)$ & Controls (\%) & OR $(95 \%$ CI $)$ & $P$ value \\
\hline \multicolumn{5}{|l|}{ rs 2230926} \\
\hline $\mathrm{TT}$ & $284(82.5)$ & 307 (89.2) & 1.00 & - \\
\hline TG & $58(16.9)$ & $36(10.5)$ & $1.74(1.12-2.72)$ & 0.015 \\
\hline GG & $2(0.6)$ & $1(0.3)$ & $2.16(0.20-23.97)$ & 0.530 \\
\hline TG/GG & $60(17.5)$ & $37(10.8)$ & $1.75(1.13-2.72)$ & 0.012 \\
\hline \multicolumn{5}{|l|}{ rs13207033 } \\
\hline GG & 277 (80.5) & $280(81.4)$ & 1.00 & - \\
\hline GA & $65(18.9)$ & $60(17.4)$ & $1.10(0.74-1.62)$ & 0.647 \\
\hline AA & $2(0.6)$ & $4(1.2)$ & $0.51(0.094-2.78)$ & 0.433 \\
\hline GA/AA & $67(19.5)$ & 64 (18.6) & $1.06(0.72-1.55)$ & 0.771 \\
\hline \multicolumn{5}{|l|}{ rs10499194 } \\
\hline $\mathrm{CC}$ & $310(90.1)$ & $325(94.5)$ & 1.00 & - \\
\hline $\mathrm{CT}$ & $34(9.9)$ & $19(5.5)$ & $1.88(1.05-3.36)$ & 0.034 \\
\hline $\mathrm{TT}$ & 0 & 0 & - & - \\
\hline
\end{tabular}

Data were calculated by unconditional logistic regression.

Table 3. Allele frequencies of polymorphisms in the TNFAIP3 gene and risk of rheumatoid arthritis in Chinese subjects

\begin{tabular}{lccccccc}
\hline $\begin{array}{l}\text { dbSNP number, } \\
\text { minor/major allele }\end{array}$ & No. of cases & No. of controls & \multicolumn{2}{c}{ Minor allele frequency } & OR (95\% CI) & P value \\
\cline { 3 - 7 } & & & cases & controls & & & \\
\hline rs2230926, G/T & 344 & 344 & 0.090 & 0.055 & $1.69(1.12-2.57)$ & 0.013 \\
\hline rs13207033, A/G & 344 & 344 & 0.100 & 0.099 & $1.02(0.71-1.45)$ & 0.928 \\
\hline rs10499194, T/C & 344 & 344 & 0.049 & 0.028 & $1.83(1.03-3.24)$ & 0.036 \\
\hline
\end{tabular}

rium in the present population. Of 8 possible haplotypes, only 3 had a frequency of $>0.03$ among both cases and controls and were included in the haplotype analysis. Three possible haplotypes represented $94.4 \%$ of the chromosomes for the cases and $90.7 \%$ for the controls. According to our a priori hypothesis and the SNP-based analyses, we considered the individuals with the rs2230926T-rs13207033Grs10499194C haplotype to be the reference group for OR estimations. The T-A-C and G-G-C haplotypes were associated with increased risks of RA (ORs were 2.46 and 2.27, $95 \%$ CIs were $1.36-4.48$ and $1.35-3.80, p$ values were 0.002 and 0.002 , respectively).

\section{Discussion}

The associations of RA susceptibility and SNPs in the intergenic region of chromosome $6 \mathrm{q} 23$ have attracted much interest in recent years $[6,7]$. Therefore, we studied the clearest candidate SNPs among the genes flanking this locus, namely TNFAIP3. Our results showed that rs10499194 and rs2230926 in the TNFAIP3 gene region were associated with risks of RA in the Chinese population and suggested that TNFAIP3 might be a common genetic risk factor for RA in Chinese subjects, which is accordant with the results in the Caucasian population [4].

The present study showed that the frequencies of the rs2230926 G allele, rs13207033 A allele and rs10499194 T allele were $0.055,0.099,0.028$ in the control group. According to Hapmap data, in the Chinese population, the prevalence of these alleles were $0.089,0.080$ and 0.011 respectively, which were similar in the present study.

Although the association between rs2230926 SNP with RA remains unclear in Caucasian patients, considering the genetic significance of rs2230926 in the Japanese patients with RA, this SNP has been included in our study. In the present study, rs2230926, located in exon 3 of TNFAIP3, was significantly associated with a predisposition to RA in Chinese subjects. SNP rs2230926 is a nonsynonymous variant that results in a phenylalanine-to-cysteine change at residue 127 of the A20 protein [11]. Musone et al. report- 
Table 4. Polymorphisms in the TNFAIP3 gene and risks of rheumatoid arthritis stratified by gender status

\begin{tabular}{|c|c|c|c|c|c|c|}
\hline \multirow[t]{2}{*}{ Genotype } & \multicolumn{3}{|c|}{ Females } & \multicolumn{3}{|c|}{ Males } \\
\hline & Cases (\%) & Controls (\%) & OR $(95 \% \mathrm{CI})$ & Cases (\%) & Controls (\%) & OR $(95 \% \mathrm{CI})$ \\
\hline \multicolumn{7}{|l|}{ rs 2230926} \\
\hline $\mathrm{TT}$ & $237(82.3)$ & 249 (88.6) & 1.00 & 47 (83.9) & $58(92.1)$ & 1.00 \\
\hline TG & $49(17.0)$ & $31(11.0)$ & $1.66(1.02-2.69)$ & $9(16.1)$ & $5(7.9)$ & $2.22(0.70-7.08)$ \\
\hline GG & $2(0.7)$ & $1(0.4)$ & $2.10(0.19-23.32)$ & 0 & 0 & - \\
\hline TG/GG & $51(17.7)$ & $32(11.4)$ & $1.67(1.04-2.70)$ & $9(16.1)$ & $5(7.9)$ & $2.22(0.70-7.08)$ \\
\hline$G$ allele & $53(9.2)$ & $33(5.9)$ & $1.62(1.04-2.55)$ & $9(8.0)$ & $5(4.0)$ & $2.12(0.69-6.51)$ \\
\hline \multicolumn{7}{|l|}{ rs13207033 } \\
\hline GG & $236(81.9)$ & $231(82.2)$ & 1.00 & $41(73.2)$ & $49(77.8)$ & 1.00 \\
\hline GA & $50(17.4)$ & 47 (16.7) & $1.04(0.67-1.61)$ & $15(26.8)$ & 13 (20.6) & $1.38(0.59-3.23)$ \\
\hline AA & $2(0.7)$ & $3(1.1)$ & $0.65(0.11-3.94)$ & 0 & $1(1.6)$ & - \\
\hline GA/AA & $52(18.1)$ & $50(17.8)$ & $1.02(0.66-1.56)$ & $15(26.8)$ & $14(22.2)$ & $1.28(0.55-2.96)$ \\
\hline A allele & $54(9.4)$ & $53(9.4)$ & $0.99(0.67-1.48)$ & $15(13.4)$ & $15(11.9)$ & $1.14\{0.53-2.46)$ \\
\hline \multicolumn{7}{|l|}{ rs10499194 } \\
\hline $\mathrm{CC}$ & $255(88.5)$ & 263 (93.6) & 1.00 & $55(98.2)$ & $62(98.4)$ & 1.00 \\
\hline $\mathrm{CT}$ & $33(11.5)$ & $18(6.4)$ & $1.89(1.04-3.44)$ & $1(1.8)$ & $1(1.6)$ & $1.13(0.07-18.45)$ \\
\hline $\mathrm{TT}$ & 0 & 0 & - & 0 & 0 & - \\
\hline $\mathrm{T}$ allele & $33(5.7)$ & $18(3.2)$ & $1.84(1.02-3.30)$ & $1(0.9)$ & $1(0.8)$ & $1.13(0.07-18.22)$ \\
\hline
\end{tabular}

Table 5. Interaction of rs 2230926 and rs 10499194 on the risk of rheumatoid arthritis in Chinese subjects

\begin{tabular}{lcccc}
\hline \multirow{4}{*}{ rs2230926 } & \multicolumn{4}{c}{ rs10499194 CC CT } \\
\cline { 2 - 5 } & Cases/Controls & OR $(\mathbf{9 5 \%} \mathbf{C I}) *$ & Cases/Controls & OR (95\% CI) \\
\hline TT & $262 / 291$ & 1.00 & $22 / 16$ & $1.53(0.79-2.97)$ \\
\hline TG/GG & $48 / 34$ & $1.57(0.98-2.51)$ & $12 / 3$ & $4.44(1.24-15.92)$ \\
\hline$*$ OR and $95 \%$ CI were calculated by logistic regression, with the rs2230926 wild genotype $(T T)$ as the reference group. \\
\hline
\end{tabular}

Table 6. Haplotype frequencies in cases and controls

\begin{tabular}{lcccc}
\hline Haplotype & Cases $(\boldsymbol{\%})$ & Controls $(\boldsymbol{\%})$ & OR $(\mathbf{9 5 \%}$ CI) & $\boldsymbol{P}$ value \\
\hline T-G-C & $564(82.0)$ & $586(85.2)$ & 1.00 & \\
\hline T-A-C & $38(5.5)$ & $16(2.3)$ & $2.46(1.36-4.48)$ & 0.002 \\
\hline G-G-C & $48(6.9)$ & $22(3.2)$ & $2.27(1.35-3.80)$ & 0.002 \\
\hline
\end{tabular}

ed that Cys127 A20 protein (encoded by the G allele) was only modestly, but consistently, less effective at inhibiting TNF-induced NF- $\kappa \mathrm{B}$ activity than the Phe127 protein (encoded by the $\mathrm{T}$ allele) [11]. This result suggests that reduced negative regulatory activity of the A20 protein may allow excessive immune activity, leading to enhanced auto reactivity. SNP rs10499194 is one of the landmark poly- morphisms identified in Caucasian patients with RA [6, 19] although the significant association with RA could not be replicated in another Caucasian population [20]. Rs10499194 was also suggested to be associated with RA susceptibility in the Japanese population [13]. Because rs10499194 is associated with RA susceptibility in our population, rs 10499194 might be a landmark for disease causal 
variants in Chinese patients with RA. However, for another SNP in the 6q23 intergenic region, rs13207033, we found no association with RA risk. This is in agreement with another study [21]. On the other hand, previous reports supported the significant association of the rs13207033 SNP with RA [6, 22]. Thus, further analysis in other sample populations will be required to confirm the association between rs13207033 and RA.

Interestingly, several signals in the TNFAIP3 gene region have recently been shown to be associated with systemic lupus erythematosus in two independent studies [11, 12]. At least three associated biomarkers were suggested in each of the two studies, although these biomarkers were not entirely consistent between each study or with the same associations reported previously in RA. Despite this disagreement, it is clear that the TNFAIP3 region plays an important role in SLE, as both studies found additional markers significantly associated with the disease including the functional exon 3 rs2230926 polymorphism. Interestingly, this variant was independently associated with RA in our study. Another recent study showed that rs10499194 is also independently associated with type 1 diabetes (T1D) [23]. Moreover, a GWAS demonstrated a strong association of TNFAIP3 with psoriasis [24]. These findings confirm the importance of the TNFAIP3 gene locus in autoimmune diseases.

TNFAIP3 may be a candidate susceptibility gene for RA because of the anti-inflammatory effects of its encoded protein. TNFAIP3 encodes a cytoplasmic zinc finger protein. The protein is required for negative regulation of the $\mathrm{NF}-\kappa \mathrm{B}$ signaling pathway, which is mediated by innate immune receptors such as TNF receptors and Toll-like receptors, and it prevents overstimulation of the innate immune response [10, 25]. Therefore, it seems likely that polymorphisms in the TNFAIP 3 gene that may reduce its protein expression or activity favor exaggerated inflammatory responses that contribute to RA development. However, our study has only provided suggestive evidence of the association of this gene with RA susceptibility. This evidence is reinforced by data from the WTCCC GWAS [22] and preliminary data presented at the 2008 American College of Rheumatology meeting [26]. Investigation of the TNFAIP3 gene region deserves increased attention owing to its likely involvement in multiple immune-related diseases and in the NF-kB pathway.

Upon further analysis, an association between the three SNPs and RA in the male subset of our population was not significant, which might due to a lack of statistical power. However, gender differences in association signals cannot be excluded yet. In the Wellcome Trust Case Control Consortium GWAS, SNP rs11761231 was suggested to be a strong signal in the gender-stratified analyses for RA, with an additive effect in females and no effect in males [22].

In conclusion, our study suggests that TNFAIP 3 might be a genetic risk factor for the development of RA in the
Chinese population. Although the SNPs rs2230926 and rs10499194 may be strong causal variant candidates in this region, a search for additional causal variants in TNFAIP3 is required.

We are grateful to patients for their participation. No funding.

The authors declare no conflict of interest.

\section{References}

1. Firestein GS (2003): Evolving concepts of rheumatoid arthritis. Nature 423: 356-361.

2. MacGregor AJ, Snieder H, Rigby AS, et al. (2000): Characterizing the quantitative genetic contribution to rheumatoid arthritis using data from twins. Arthritis Rheum 43: 30-37.

3. Alarcon-Segovia D, Alarcon-Riquelme ME, Cardiel MH, et al. (2005): Familial aggregation of systemic lupus erythematosus, rheumatoid arthritis, and other autoimmune diseases in 1,177 lupus patients from the GLADEL cohort. Arthritis Rheum 52: 1138-1147.

4. Gregersen PK, Olsson LM (2009): Recent advances in the genetics of autoimmune disease. Annu Rev Immunol 27: 363-391.

5. Bowes J, Barton A (2008): Recent advances in the genetics of RA susceptibility. Rheumatology (Oxford) 47: 399-402.

6. Plenge RM, Cotsapas C, Davies L, et al. (2007): Two independent alleles at $6 \mathrm{q} 23$ associated with risk of rheumatoid arthritis. Nat Genet 39: 1477-1482.

7. Thomson W, Barton A, Ke X, et al. (2007): Rheumatoid arthritis association at 6q23. Nat Genet 39: 1431-1433.

8. Wertz IE, O'Rourke KM, Zhou H, et al. (2004): De-ubiquitination and ubiquitin ligase domains of A20 downregulate NFkappaB signalling. Nature 430: 694-699.

9. Boone DL, Turer EE, Lee EG, et al. (2004): The ubiquitinmodifying enzyme A20 is required for termination of Toll-like receptor responses. Nat Immunol 5: 1052-1060.

10. Lee EG, Boone DL, Chai S, et al. (2000): Failure to regulate TNF-induced NF-kappaB and cell death responses in A20deficient mice. Science 289: 2350-2354.

11. Musone SL, Taylor KE, Lu TT, et al. (2008): Multiple polymorphisms in the TNFAIP3 region are independently associated with systemic lupus erythematosus. Nat Genet 40 : 1062-1064.

12. Plenge RM, Cotsapas C, Davies L, et al. (2007): Two independent alleles at $6 \mathrm{q} 23$ associated with risk of rheumatoid arthritis. Nat Genet 39: 1477-1482.

13. Shimane K, Kochi Y, Horita T, et al. (2010): The association of a nonsynonymous single-nucleotide polymorphism in TNFAIP3 with systemic lupus erythematosus and rheumatoid arthritis in the Japanese population. Arthritis Rheum 62: 574579.

14. Arnett FC, Edworthy SM, Bloch DA, et al. (1988): The American Rheumatism Association 1987 revised criteria for the classification of rheumatoid arthritis. Arthritis Rheum 31: 315324.

15. Kochi Y, Okada Y, Suzuki A, et al. (2010): Regulatory variant in CCR6 is associated with rheumatoid arthritis susceptibility. Nat Genet 42: 515-519.

16. Lee HS, Korman BD, Le JM, et al. (2009): Genetic risk factors for rheumatoid arthritis differ in caucasian and Korean populations. Arthritis Rheum 60: 364-371. 
17. Shi YY, He L (2005): SHEs is, a powerful software platform for analyses of linkage disequilibrium, haplotype construction, and genetic association at polymorphism loci. Cell Res 15: 97-98.

18. Li Z, Zhang Z, He Z, et al. (2009): A partition-ligationcombination-subdivision EM algorithm for haplotype inference with multiallelic markers: update of the SHEsis (http://analysis.bio-x.cn). Cell Res 19: 519-523.

19. Orozco G, Hinks A, Eyre S, et al. (2009): Combined effects of three independent SNPs greatly increase the risk estimate for RA at 6q23. Hum Mol Genet 18: 2693-2699.

20. Perdigones N, Lamas JR, Vigo AG, et al. (2009): 6q23 polymorphisms in rheumatoid arthritis Spanish patients. Rheumatology (Oxford) 48: 618-621.

21. Dieguez-Gonzalez R, Calaza M, Perez-Pampin E, et al. (2009): Analysis of TNFAIP3, a feedback inhibitor of nuclear factorkappaB and the neighbor intergenic $6 \mathrm{q} 23$ region in rheumatoid arthritis susceptibility. Arthritis Res Ther 11: R42.

22. Wellcome Trust Case Control Consortium, et al. (2007): Genome-wide association study of 14,000 cases of seven common diseases and 3,000 shared controls. Nature 447: 661-678.

23. Fung E, Smyth DJ, Howson JM, et al. (2008): Analysis of 17 autoimmune disease-associated variants in type 1 diabetes identifies 6q23/TNFAIP3 as a susceptibility locus. Genes Immun 10: 188-191.

24. Nair RP, Duffin KC, Helms C, et al. (2009): Genome-wide scan reveals association of psoriasis with IL-23 and NF-kappaB pathways. Nat Genet 41: 199-204.

25. Liu YC, Penninger J, Karin M (2005): Immunity by ubiquitylation:a reversible process of modification. Nat Rev Immunol 5: 941-952.

26. Thomson W, Barton A, Eyre S, et al. (2008): Characterization of the $6 \mathrm{q} 23$ rheumatoid arthritis susceptibility locus. Arthritis Rheum 58: S214-S215. 